\title{
ADVANCED DISTRIBUTION MANAGEMENT SYSTEM
}

\author{
Artur R. Avazov ${ }^{1,}$, Liubov A. Sobinova ${ }^{1}$ \\ ${ }^{1}$ National Research Tomsk Polytechnic University, 634050 Tomsk, Russia
}

\begin{abstract}
This article describes the advisability of using advanced distribution management systems in the electricity distribution networks area and considers premises of implementing ADMS within the Smart Grid era. Also, it gives the big picture of ADMS and discusses the ADMS advantages and functionalities.
\end{abstract}

\section{Introduction}

The twenty first century has marked by big technological progress. With the development of new technologies, pressing issue, related to the reequipment of the energy sector has become more prominent. The implementing of up-to-date electricity-powered devices such as battery-powered vehicles and high-tech equipment has increased the energy demand, but existing engineering management tools can not provide required level of reliability and safety of the energy distribution process. There are several cases able to explain the current situation:

1. Aging workforce and infrastructure;

2. Absence of wide-dimension software which would provide different functionalities unified in one program solution;

3. Modern electricity equipment implementing;

4. Appearance of distributed generation.

To solve the above referenced problems the smart grid technologies and ADMS are being increasingly developed. The ADMS (advanced distribution management system) technology and its features will be described in this white paper.

For more simplified understanding of considered issue, there are Smart grid related acronyms, listed in Table 1.

Table 1. Smart Grid related acronyms.

\begin{tabular}{|l|l|}
\hline Acronym & Definition \\
\hline AMI & Automatic metering infrastructure \\
\hline DLC & Direct load control \\
\hline DMS & Distribution management system \\
\hline DR & Demand response \\
\hline EMS & Energy management system \\
\hline OMS & Outage management system \\
\hline SCADA & Supervisory control and data acquisition \\
\hline
\end{tabular}

\section{a Corresponding author: mad2@tpu.ru}




\section{Problem statement}

What is an ADMS? "An advanced distribution management system (ADMS) is the software platform that supports the full suite of distribution management and optimization. An ADMS includes functions that automate outage restoration and optimize the performance of the distribution grid. ADMS functions being developed for electric utilities include fault location, isolation and restoration; volt/volt-ampere reactive optimization; conservation through voltage reduction; peak demand management; and support for microgrids and electric vehicles." - Gartner IT Glossary.

The ADMS is a decision support system that helps control room and field operating personnel to monitor and control the electric distribution system effectively while improving safety, reliability, asset protection and quality of service. ADMS delivers a single environment and user experience. This enables the streamlining of decision making and enhances emergency response performance. It resolves the critical barrier issues of real-time integration and enables the creation of highperformance network models. By providing a unified environment for control and dispatch, ADMS also allows for a more comprehensive view of the distribution system during an outage. The Advanced DMS is the utility depended technology that is why it should be interconnected with the existing Energy Management or Network Management System and OMS. Data sharing between these systems maximizes the efficiency of operation within your control room or control centers.
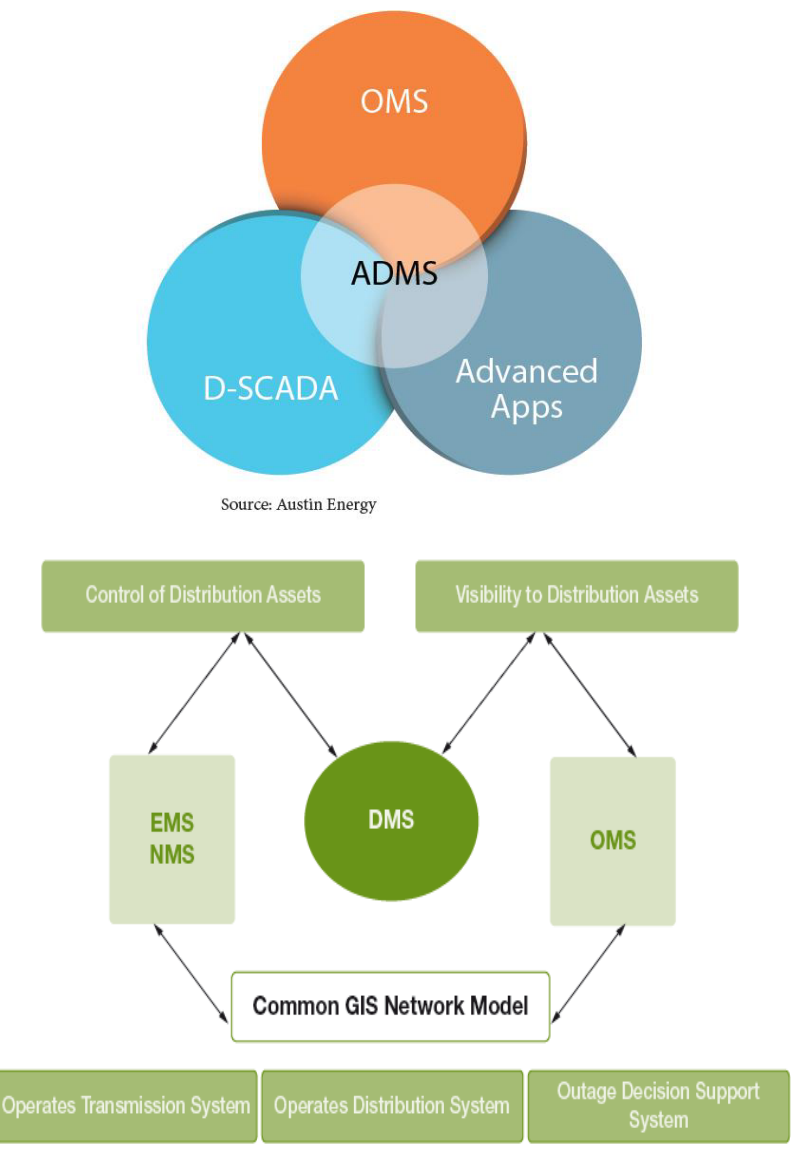

Figure 1. The structure of distribution management system.

Moreover, with the increase of energy consumers it is mandatory to enlarge the hardware structure in order to keep up the processed data. For this reason, new open structures are being implemented with ADMS. It allows increasing the accuracy level of the system analysis, data calculation and network representation. 
The ADMS includes monitoring, analysis, control, optimization, training and planning tools that all function on a common representation of the entire electric distribution network. This allows system operators, dispatchers, reliability analysts, planning engineers and managers to work as a team accessing the same as-operated representation of network information for efficient and reliable management of grid operations.

With the ADMS there is the possibility to visualize the current state of network based on SCADA telemetery combined with state estimation for all non-telemetered equipment to provide systems operators with greater network awareness.

Owing to this modern technology it is possible to accurately model all elements of the network for better load forecasting, fault location prediction, energy loss reduction and equipment failure prevention. It provides a proven SCADA infrastructure, outage management solution and the most advanced analysis functions on the market. All in one comprehensive management solution. ADMS turns data into actionable business intelligence to increase the efficiency and support decisions across entire enterprise. Also ADMS allows to improve the safety and security field operations for planned and unplanned network events through high-quality simulations of switching and restoration activities before operating devices and dispatching field personnel. In addition, it provides superior reliability and customer satisfaction with self-healing, closed loop control capabilities. It delivers significant functionality to manage outages and perform automated fault location, isolation and supply restoration operations.

Besides, ADMS supports distributed generation enabling end-user utility to grow and accommodate for renewable energy of the smart grid era, well optimizing the network for reliable service. It also supports near, short, medium and long-term load forecasting for network planning and an extensive training simulator. With ADMS the network can be optimized with integrated Volt/VAR optimization, improving power quality in all parts of the distribution network. Volt/VAR capabilities also provide a transparent approach to voltage based demand response functionality which can be exercised without impact on customers.

The ADMS benefits:

- Demand management;

- Energy loss prevention;

- Peak shaving;

- Reduced peak power purchases;

- Improved operational efficiency

- Support for regulatory compliance

- Better assets utilization

- Standards-based integration

Furthermore, these benefits can contribute to significant savings for utilities and customers.

The foundation of the ADMS

The major ADMS capability is to accurately define the network model and to process an unbalanced load flow algorithm, based upon this model with telemetered data, acquired from the network.

- The network model

The ADMS must be able to represent all aspects of the distribution networks, including a variety of conductor types, transformers, switches, fuses, etc. and monitor the status of these ones.

- The dynamic data

In order to make ADMS load flow algorithm more exact it is necessary to provide telemetered data from distribution network. Data is usually obtained from SCADA (Supervisory Control and Data Acquisition) systems. The AMI (advanced metering infrastructure) and OMS (Outage Management) are subsystems, which allow assembling and transferring data. ADMS requires a lot of data which changes quickly. For this reason the high-performance data management is indispensable. This data provides a lot of information (Voltage, current) and device status to enable the load flow algorithm to promote more reliable results.

- The unbalanced load flow algorithm 
The ADMS must have a significantly fast load flow algorithm to solve unbalanced distribution networks.

Main ADMS components

- Control system

The ADMS typically includes a SCADA system, an OMS and advanced applications.

- Data is significantly important

The ADMS requires data to understand the full potential of its function. Data is gathered from intelligent field devices and enterprise systems. Besides, AMI grids can render the last gasp information to enforce outage management processing within ADMS as well as the capability to perform output meter pings to test for voltage potential at the meter base endpoint.

- Mobility

Mobile systems can be used to directly view and operate the network on mobile data terminals. OMSs get enforced abilities through mobility by implementing the digital ticket system for team dispatch and tracking, occurrence/cause/repair reporting and up to date customer outage information.

- Advanced applications

The next point is a description of available advanced applications.

- Relay Protection Analysis, Adaptive Protection, Closed Loop

This applications are interconnected with each other. The relay protection analysis application accomplishes analytical studies on the network. If a system is complemented by adaptive protection, it can determine the correct relay setting to support new configurations. As soon as the new configuration is recognized with a closed loop application, the system will automatically reconfigure without the need for operator interruption.

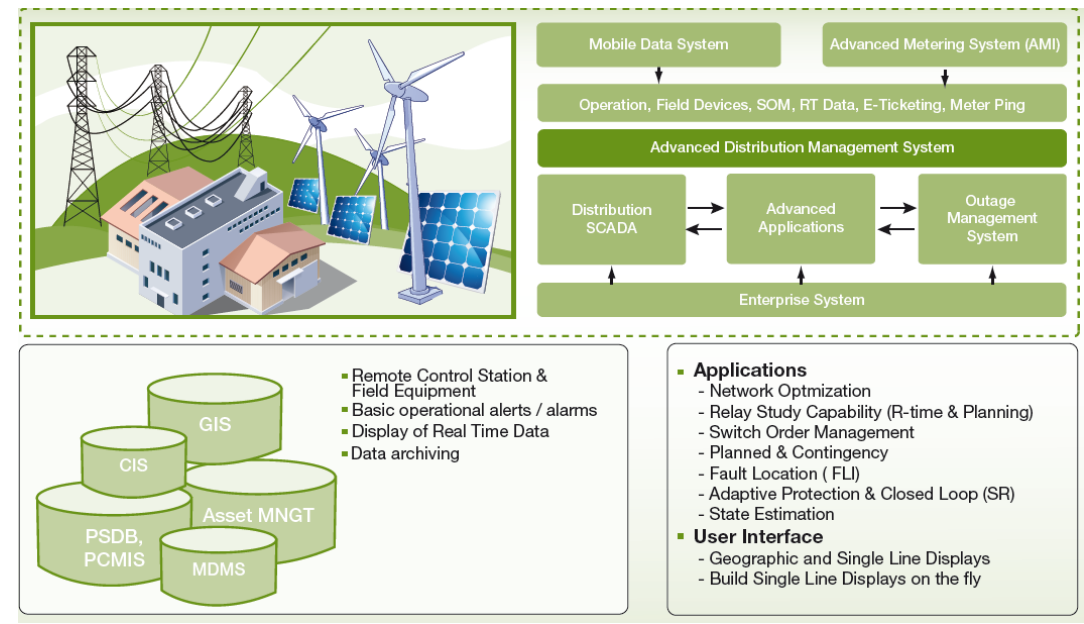

Figure 2. Main components.

- Switch order management

This application provides the user with the option to create switching orders manually or automatically. The application allows the user to customize the switching order to reflect current inhouse templates. The business benefit is that all relevant factors are being analyzed on a single platform providing improved safety, reliability and operating effectiveness.

- Fault Location Isolation Service Restoration (FLISR)

This application enhances the Fault Location process. Customer restoration is improved by having better information and the ability to direct field crews more accurately to the faulted location. Using information obtained from the relay IED and fault detectors location in the field will avoid lengthy line patrols and sectionalizing efforts. Some DMS solutions also provide means whereby data administrators can input historical failures for the feeder in the application. This historical information 
can be used as one of the mechanisms for determining faulted locations as well. The Isolation and Service Restoration piece of FLISR is the next step. Once the faulted section is known, remotely controlled devices can restore customer load either manually (via remote control) or automatically (without operator intervention).

The business benefits include: improved field resource utilization, reduced customer interruptions and less "windshield time" for field forces.

- Topology Analyzer

The Topology Analyzer application displays voltages, phasing, the state of the system (energized or de-energized) using different color schemes as determined by the utility. This application is applicable to the single line schematic displays and geographic displays. Some DMS solutions can create single line displays on the fly by the user selecting the feeders and substations they wish displayed in isolation of other superfluous equipment. From a user perspective this is very powerful as it provides simplicity and clarity for the task at hand.

The business benefit is somewhat obvious. Without a method to simplify the electrical connectivity, the visual diagrams would be extremely cluttered and not useful. A lot of work has been done by the vendor community to ensure optimum flexibility provided for the user.

The ADMS's analytical functionality.

The ADMS has the multifaceted functions. In this part it should be mentioned about some of them.

- Volt/VAR Optimization (VVO)

The Volt/VAR Optimization (VVO) application in its simplest form reduces the distribution system voltage, thereby reducing the overall demand. Today, there are a number of devices that can be utilized to manage the network at a lower voltage profile, including:

- Substation - under load tap changers

- Capacitors - large and small

- Distribution stations - under load tap changers

- Other DER devices

The VVO application has been designed to optimize the use of all of the mentioned devices to minimize the overall load on the system. Typically the VVO applications are rule-based to determine the order and use of these devices.

The business benefit is the reduction of power consumption. This will offset the need for capital upgrades such as additional generating facilities and network upgrades to meet increased load demands.

- Network reconfiguration

This application allows network optimization in case of new devices/customers deployment, fault or power imbalance occurrence. The objectives are minimizing line losses, providing optimum phase balancing while maintaining adequate voltage profiles.

- State estimation

In power network state estimation validates observable data and calculates all non-telemetered points in order to derive a complete network view.

The collected data quality is not very precise due to problems in devices or telecommunication networks. To solve this issue and provide an advanced analysis, the obtained data must be preprocessed in order of elimination of bad data points.

Distribution state estimation is much more advanced than a simple load calibration. For this reason the distribution state estimation should have the highest precision as it is the basis of all advanced functions.

- Peak shaving

Near-term load forecasting is a great tool to identify the potential for a peak load event during the course of the day. Combining this forecast capability with Volt/VAR Optimization is a great way to continue managing the demand on the network without affecting any customers. Load tap changers, voltage regulators, and capacitors can be automatically or manually controlled to maintain voltage profiles, within regulatory limits, in order to manage demand. This can be done only during peak events or as a normal mode of operation as desired. 


\section{Results and discussion}

Demand response is the main function that drives utilities to Smart Grid programs and to the implementation of advanced DMS software. The complex system of demand response offers a lot of options that can be accomplished to reduce demand in case of increasing demand, and the potential of costs rising and network instability.

Some demand response actions depend on customers to react on their own. Utilities operationally enquire for electricity consumers to change their consumption patterns when supply is short. Regulators are also able to create rate structures that encourage similar conduct through normal attention to energy costs. However, since these approaches do not provide enough reduction, additional demand response options are rendered, and the ADMS can support the prioritized application of these options.

Generally, the available options are divided into three categories, which are listed in the dependence of customer impact (lowest to highest):

1.Conservation voltage management (CVR) - also known as Distribution System Demand Response (DSDR)

This option includes functionality of volt/VAR management in order to reduce demand by means of voltage reduction on the distribution network. The voltage reduction drives in reduction in the power which is delivered by a similar amount without customer interruption. Moreover, voltage management can be accomplished on a feeder or feeder segment basis to resolve a local over load (perhaps as a result of electric vehicles or other infrequent demand anomalies) instead of on a system wide basis.

2. Direct load control

Most utilities have implemented some form of direct load demand response, generally through radio-controlled devices located on water heaters, air conditioners and pool pumps. Using this form of demand response, utilities can turn off these devices for a short period of time (perhaps 15 to 20 minutes) to reduce demand on the distribution network. The direct load programs already in place rotate the "turn-offs" around the service territory to reduce customer impact. Since there is some amount of customer impact, while minimal, utilities must generally ask customers to "opt-in" to such a program, and often trade a rate advantage or discount to customers who choose the program.

Direct load control will continue to increase in complexity with the evolution of home area network (HAN) technology and the continued roll-out of AMI systems. The advanced DMS is designed for integration with these end-of-network systems, supporting direct load control by identifying appropriate feeders segments and timing in a prioritized manner.

\section{Load interruption/islanding}

When other demand response options fail, utilities disconnect some demand to maintain the ability to serve the rest. Again, most utilities have "opt-in" programs for such interruption, including interruptible rates that are supported by regulators and governments. However, depending on the nature of the overload and danger to the health of the distribution network, utilities may have to exercise interruption options with little or no advance notice - even to customers who have not elected this option. Once again, the advanced DMS makes recommendations and prioritizes options for utility dispatchers to enable decision making in situations where it is unavoidable.

An ADMS platform provides a new future for grid and outage management by providing a single environment and user experience for SCADA, DMS and OMS. This key construct resolves the critical barrier issues of real-time integration and high-performance network models. By providing a unified environment for control and dispatch, ADMS allows for a more comprehensive view of the distribution system during an outage. Placing the tools for outage analysis and crew dispatch alongside those for control, load flow, and grid optimization enables a workflow that is more responsive and less error-prone.

ADMS also eliminates the network modeling problem, enabling OMS functionality against the memory-resident, real-time model of SCADA / DMS. A single, high-performing network model for SCADA, DMS, and OMS improves accuracy and performance, and eliminates the need for data 
synchronization among disparate models. The concept, also known as „The Single Version of the Truth $^{\text {ee }}$ is a huge step forward in both OMS performance and enterprise data integrity.

ADMS provides the user access to a robust variety of network analytics to assist in outage management and service restoration. Decision support functions, such as fault location, can reduce patrol time - limiting unnecessary truck travel and shortening outage times. Large area load restoration functions can provide dispatchers with simplified scenarios for bringing back online large sections of the network in the event of a wide-spread grid failure. Switch management functionality along with a fast load flow solution lets operators quickly analyze pre-defined switching scenarios in the context of current conditions. This avoids overloads and repeated re-energizing of lines that shorten the lifecycle of cables and oil-filled equipment.

Operations control center employees are under pressure to make decisions quickly. Too much information undermines workflow as much as not enough information. ADMS presents data and function in a simple yet thorough way which allows operators to perform advanced outage management.

\section{Conclusion}

Today, the utility industry is rallying behind the Smart Grid as a way to make valuable infrastructure improvements, increase customer options, and improve efficiency. The Smart Grid requires utilities to seriously consider an Advanced Distribution Management System to accommodate and optimize the new technology that will be installed in the field. ADMS solutions are needed to analyze the massive amounts of new data generated by Smart Grid devices, perform automated tasks to support applications like Volt/VAR and FLISR in a closed loop providing new levels of operating effectiveness and work load management improvement in the control room.

Finally, ADMS speeds cost recovery for Smart Grid investments, offsets the need to construct new central generation, and provides a flexible grid-management platform that can accommodate emerging demands from the utility customer, shareholder and regulator.

\section{References}

1. P.E. John Dirkman, Enhancing utility outage management system, Schneider Electric, 6 (2014)

2. Telvent utilities group. The Advanced Distribution Management System (ADMS). Schneider Electric, Whitepaper (2012)

3. Advanced Distribution Management Systems, Capgemini Consulting (2012)

4. Wenye Wang, Yi Xu, Mohit Khanna. A survey on the communication architectures in smart grid. Survey paper. 